\title{
Comparison of Bromocriptine and Hydroxyethyl Starch for Reducing Ohss in in Vitro Fertilization Patients: A Retrospective Study
}

\section{Qiaoli Zhang}

Capital Medical University Beijing Obstetrics and Gynecology Hospital

\section{Yanmin Ma}

Capital Medical University Beijing Obstetrics and Gynecology Hospital

\section{Xiaomeng Bu}

Capital Medical University Beijing Obstetrics and Gynecology Hospital

Chanwei Jia

Capital Medical University Beijing Obstetrics and Gynecology Hospital

Yanjun Liu

Capital Medical University Beijing Obstetrics and Gynecology Hospital

Shuyu Wang ( $\nabla$ wangshuyu@ccmu.edu.cn )

Capital Medical University Beijing Obstetrics and Gynecology Hospital

\section{Research}

Keywords: Ovarian hyperstimulation syndrome, Bromocriptine, Hydroxyethyl Starch, Prevention, In-vitro fertilization

Posted Date: April 12th, 2021

DOl: https://doi.org/10.21203/rs.3.rs-384742/v1

License: (c) (i) This work is licensed under a Creative Commons Attribution 4.0 International License. Read Full License 


\section{Abstract}

Background: The ovarian hyperstimulation syndrome (OHSS) is a potentially life-threatening complication associated with controlled ovarian hyperstimulation (COS) during assisted reproductive technology (ART) treatment, and effective preventive measures are urgently needed. The dopamine agonist can mitigate OHSS incidence through a decreased vascular permeability via reduced vascular endothelial growth factor (VEGF) production. This study evaluated the clinical effectiveness of bromocriptine, an alternative DA, for prevention of OHSS in high risk women.

Methods: The retrospective study population consisted of women undergoing in vitro fertilization (IVF) or intracytoplasmic sperm injection (ICSI) cycles with GnRH antagonist protocols, at risk of OHSS. The study group included 52 women given $2.5 \mathrm{mg}$ bromocriptine daily by rectal insertion for 5 days beginning on the day of oocyte retrieval; the control group included 52 women given $500 \mathrm{ml}$ intravenous (I.V.) hydroxyethyl starch (HES) daily for 5 days also beginning on the day of oocyte retrieval. The outcomes of ovarian stimulation, incidence and severity of OHSS, blood-related indicators, biochemical indicators (liver and renal function), and coagulation and fibrinolytic activities were compared.

Results: The groups were not significantly different in age, estradiol concentration on the day of human chorionic gonadotropin injection, or number of retrieved oocytes. The occurrence of mild $(13.46 \%$ vs $15.48 \%$ ) and moderate (7.69\% vs $5.77 \%$ ) OHSS were not significantly different between bromocriptine and HES groups ( $P>0.05)$. No cases of severe or late OHSS were recorded in either group. Only hemocratin levels were significantly higher and activated partial thromboplastin time was significantly lower in the bromocriptine group $(P<0.05)$ compared to the HES group on Day 5 after oocyte retrieval. No difference in liver or renal function was found between groups.

Conclusions: Prophylactic rectal administration of bromocriptine was as effective as I.V. HES for prevention of OHSS.

\section{Introduction}

Assisted reproductive technology (ART) therapy may be accompanied by the development of ovarian hyperstimulation syndrome (OHSS), a complication that is connected to controlled ovarian hyperstimulation (COS), which can be potentially fatal in some circumstances. During OHSS, the ovaries become enlarged and an acute shift in fluids occurs from the intravascular to the third space, sometimes resulting in fluid accumulation in the peritoneal and thoracic cavities, with subsequent hemoconcentration, elevated hematocrit, and decreased organ perfusion [1-3]. Kidney failure, respiratory distress, and death are all complications associated with OHSS[4].

As much as $20-33 \%$ of in vitro fertilization (IVF) cycles may be affected by mild OHSS, whereas moderate to severe OHSS can reportedly occur in $3 \%$ to $8 \%$, respectively, during ART cycles, the proportions are likely to be substantially higher among women with polycystic ovaries, high oocyte yield from OPU, or other high risk factors [5]. Some studies have proposed that OHSS-related fluid accumulation may 
develop in high-risk women due to greater vascular permeability associated with stimulation by ovarian steroids and treatment with human chorionic gonadotropin (hCG). Moreover, several hormones and factors have been identified as potential etiological agents in OHSS, including prostaglandins, inhibin, the renin-angiotensin-aldosterone system, as well as several inflammatory factors [6], with vascular endothelial growth factor (VEGF) serving as the primary mediator of this syndrome $[7][2,8]$.

A systematic literature review and meta-analysis revealed that prophylactic administration of dopamine agonist (DA) can lead to significantly reduced incidence of OHSS among high-risk patients, most substantially so among early onset cases [9]. A growing number of studies have evaluated cabergoline as a pharmacological agent to reduce OHSS incidence and severity. However, cabergoline is unavailable in many parts of the world, including mainland China. An alternative DA, bromocriptine, which is widely available, has also been previously reported to attenuate OHSS, but has received less research attention [10]. This study aimed to compare the prophylactic effectiveness of rectal bromocriptine administration with that of hydroxyethyl starch (HES) infusion in the prevention of OHSS among high risk women receiving ART.

\section{Materials And Methods}

\section{Subject recruitment}

This retrospective study was performed at the Department of Human Reproductive Medicine, Beijing Obstetrics and Gynecology Hospital, Capital Medical University, Beijing. We enrolled 104 infertile women at high risk of developing OHSS from among the patient population with IVF or intracytoplasmic sperm injection (ICSI) cycles between February 2019 and December 2020 in our clinic. "High risk of OHSS" was defined as serum estradiol $\left(E_{2}\right)$ levels $>3000 \mathrm{pg} / \mathrm{ml}$ at the time of trigger, and/or retrieval of 20 or more oocytes [11]. The exclusion criteria were patients with a history of cancer, only one ovary, as well as coasting cases. The Institutional Review Board and Ethical Committee of Beijing Obstetrics and Gynecology Hospital, Capital Medical University, Beijing approved this retrospective study.

\section{Protocols for ovarian stimulation}

All patients (25-39 years old) underwent ovarian stimulation using a protocol that combined recombinant follicle stimulating hormone ( $\mathrm{rFSH}$ ) (Gonal F® Merck, Modugno, Italy), highly purified urinary menopausal gonadotropins (HP-HMG) (Menopur ${ }^{\circledR}$, Kiel, Germany), Menotrophin (Ferti® M, Livzon, Zhuhai, China), and gonadotrophin releasing hormone $(\mathrm{GnRH})$ antagonist (Cetrotide ${ }^{\circledR} 0.25 \mathrm{mg}$ Merck, Lyon, France ). The initial dose was individualized based on patient age, ovarian reserve tests, body mass index (BMI), and subsequent doses were tailored according to her response after the first 5 days of stimulation [12]. Follicular development was monitored by transvaginal ultrasound (TVS) and serum $\mathrm{E}_{2}, \mathrm{P}$, and LH levels (measured by chemiluminescence). When an echography revealed at least three follicles $>18 \mathrm{~mm}$ in diameter, $0.2 \mathrm{mg} \mu \mathrm{g}$ GnRH Agonist (Decapeptyl®, $0.1 \mathrm{mg}$, Ferring, Kiel, Germany) was administered 
subcutaneously. Transvaginal ultrasound-guided oocyte retrieval was then scheduled for $36 \mathrm{~h}$ after the trigger.

\section{OHSS preventive treatment administration}

One-hundred and four patients at high risk of OHSS were eligible: the study group $(n=52)$ received 2.5 $\mathrm{mg}$ bromocriptine through daily rectal administration for 5 days, starting on the day of oocyte retrieval (i.e., Day 0$)$, while the control group $(n=52)$ received a slow intravenous (I.V.) infusion of $500 \mathrm{ml} \mathrm{HES}$ daily for 5 days, beginning on the day of oocyte retrieval.

\section{Assessment of ovarian hyperstimulation syndrome}

OHSS was diagnosed and classified as previously described by Humaidan et al [13]. Patients were assessed for clinical symptoms, signs, and laboratory data of OHSS at 5 days after oocyte retrieval. "early OHSS" was defined as occurring $\leq 9$ days after oocyte retrieval, while presentation of OHSS later than 9 days was classified as "late OHSS" [14].

In both groups, hematological tests were performed before ovarian stimulation (i.e., baseline) and on the 5th day after oocyte retrieval (i.e., Day 5) to determine the white blood cell (WBC) and platelet (PLT) counts, hematocrit (HCT) content, liver and renal function, and coagulation and fibrinolytic parameters.

\section{Statistical analysis}

Statistical analysis was performed using SPSS (Version 16, SPSS Inc., Chicago, IL, USA). Results of the study were expressed as means \pm standard deviation (S.D.) or number (percentage). Chi-square test or student $t$-test were performed to evaluate the statistical differences between the variables. $P<0.05$ was considered significant.

\section{Results}

\section{Baseline characteristics}

The patients' baseline characteristics and clinical features are shown in Table 1. There were no significant differences between the two groups with regard to age, BMI, duration of infertility, basal serum follicle stimulating hormone (FSH) levels, basal luteinizing hormone (LH) levels, basal $E_{2}$ levels, or type of infertility. In addition, their characteristics related to OHSS risk factors, including ovarian antral follicle count (AFC) and anti-Müllerian hormone (AMH) levels were also similar between in the study or control groups (Table 1).

Table 1 Baseline characteristics and clinical features of patients in two groups 


\begin{tabular}{|llll|}
\hline Variables & Bromocriptine $(\boldsymbol{n}=\mathbf{5 2})$ & I.V HES $(\boldsymbol{n}=\mathbf{5 2})$ & $P$-value \\
\hline Age $(\mathrm{y})$ & $31.31 \pm 3.85$ & $29.68 \pm 3.06$ & 0.093 \\
\hline $\mathrm{BMI}\left(\mathrm{kg} / \mathrm{m}^{2}\right)$ & $22.36 \pm 2.33$ & $24.07 \pm 4.15$ & 0.239 \\
\hline Duration of infertility $(\mathrm{y})$ & $2.33 \pm 1.15$ & $3.14 \pm 2.03$ & 0.216 \\
\hline Basal FSH $(\mathrm{mlU} / \mathrm{ml})$ & $6.20 \pm 1.72$ & $6.00 \pm 1.18$ & 0.638 \\
\hline Basal $\mathrm{LH}(\mathrm{mlU} / \mathrm{ml})$ & $5.01 \pm 4.05$ & $5.79 \pm 2.35$ & 0.412 \\
\hline Basal $\mathrm{E}_{2}(\mathrm{pg} / \mathrm{ml})$ & $48.49 \pm 14.71$ & $44.97 \pm 14.11$ & 0.403 \\
\hline Basal AMH $(\mathrm{ng} / \mathrm{ml})$ & $8.38 \pm 5.37$ & $9.52 \pm 4.62$ & 0.125 \\
\hline antral follicle count $(\mathrm{AFC})$ & $18.09 \pm 4.25$ & $19.10 \pm 3.30$ & 0.150 \\
\hline Type of infertility & & & \\
\hline Primary infertility, $\mathrm{n}(\%)$ & $27(51.92)$ & $29(55.77)$ & 0.187 \\
\hline Secondary infertility, $\mathrm{n}(\%)$ & $25(48.08)$ & $23(44.23)$ & \\
\hline
\end{tabular}

Note: Values represent means \pm standard deviation (SD) or number (percentage)

\section{Ovarian stimulation outcomes}

No significant differences were found between the rectal bromocriptine administration or I.V. HES groups in terms of their duration of ovarian stimulation in days ( $P>0.05)$, total amount of gonadotropin $(\mathrm{Gn})$ dose ( $P>0.05$ ), and endometrial thickness on the day of trigger ( $P>0.05)$. The subjects' characteristics were also similar between groups in relation to OHSS-associated risk factors including $E_{2}$ levels on the day of trigger, on the day of ovum pick-up (OPU), and on the 5th day after oocyte retrieval, as well as in the number of retrieved oocytes (Table 2 ).

Table 2 Ovarian stimulation outcomes in two groups 


\begin{tabular}{|llll|}
\hline Variable & $\begin{array}{l}\text { Bromocriptine } \\
(\mathbf{n = 5 2})\end{array}$ & I.V HES $(\mathbf{n}=52)$ & $P$-value \\
\hline Stimulation length $(\mathrm{d})$ & $10.27 \pm 1.74$ & $9.61 \pm 1.99$ & 0.346 \\
\hline Gn dose $(\mathrm{IU})$ & $2059.09 \pm 304.40$ & $2057.14 \pm 552.45$ & 0.991 \\
\hline $\mathrm{E}_{2}$ on the day of trigger $(\mathrm{pg} / \mathrm{ml})$ & $5811.88 \pm 1940.20$ & $6083.99 \pm 2159.79$ & 0.245 \\
\hline LH on the day of trigger $(\mathrm{mlU} / \mathrm{ml})$ & $1.35 \pm 1.11$ & $1.83 \pm 2.21$ & 0.321 \\
\hline P on the day of trigger $(\mathrm{ng} / \mathrm{ml})$ & $1.11 \pm 0.42$ & $1.25 \pm 0.63$ & 0.165 \\
\hline$E_{2}$ on Day 0 $(\mathrm{pg} / \mathrm{ml})$ & $3170.38 \pm 1157.99$ & $3538.36 \pm 1438.60$ & 0.329 \\
\hline$E_{2}$ on Day $5(\mathrm{pg} / \mathrm{ml})$ & $3209.22 \pm 1724.45$ & $2869.71 \pm 2001.64$ & 0.452 \\
\hline $\begin{array}{l}\text { Endometrial thickness on day of trigger } \\
\text { (mm) }\end{array}$ & $10.80 \pm 2.22$ & $10.67 \pm 2.21$ & 0.873 \\
\hline No. of retrieved oocytes $(\mathrm{n})$ & $23.89 \pm 4.01$ & $24.75 \pm 3.06$ & 0.061 \\
\hline
\end{tabular}

Note: Values represent means \pm standard deviation (SD)

Day 0: on the day of ovum pick-up (OPU)

Day 5: on the 5th day after oocyte retrieval

\section{OHSS Outcomes}

o significant differences were observed in the incidence of mild or moderate OHSS between the bromocriptine and HES groups. Moderate OHSS was observed in 4 patients in the bromocriptine group, while 3 cases occurred in the HES group (7.69\% vs $5.77 \%)$, with no significant differences in the two groups. These cases were all categorized as "early OHSS", and no severe OHSS or late OHSS were observed in either the study or control groups (Table 3).

Table 3 Incidence of OHSS in patients given bromocriptine or HES

\begin{tabular}{|llll|}
\hline & Bromocriptine $(\boldsymbol{n = 5 2 )}$ & I.V HES $(\boldsymbol{n}=52)$ & $P$-value \\
\hline Mild OHSS, $\mathrm{n}(\%)$ & $7(13.46)$ & $8(15.38)$ & 0.78 \\
\hline Moderate OHSS, $\mathrm{n}(\%)$ & $4(7.69)$ & $3(5.77)$ & 0.69 \\
\hline Overall OHSS, $\mathrm{n}(\%)$ & $11(21.15)$ & $11(21.15)$ & 1.00 \\
\hline Early OHSS, $\mathrm{n}(\%)$ & $11(21.15)$ & $11(21.15)$ & 1.00 \\
\hline
\end{tabular}

Note: Data are expressed as number (percentage) 
There were no significant differences found between the two groups in their baseline HCT, WBC, and PLT counts (i.e., before ovarian stimulation). However, on the 5th day after oocyte retrieval, WBC counts were significantly higher in both groups compared with their baseline WBC $(P<0.05)$. Furthermore, WBC count and HCT were both significantly higher in the bromocriptine group compared to the HES group $(P<0.05)$ on the 5th day after oocyte retrieval (Table 4).

Table 4 Blood-related parameters before ovarian stimulation and on the 5th day after oocyte retrieval

\begin{tabular}{|lll|}
\hline Variable & Bromocriptine $(n=52)$ & I.V. HES $(n=52)$ \\
\hline HCT $(\%)$ & & \\
Baseline & $40.23 \pm 2.42$ & $40.00 \pm 2.35$ \\
\hline On Day 5 & $41.43 \pm 1.93^{\star}$ & $39.52 \pm 3.29$ \\
\hline WBC count $\left(\times 10^{9} / L\right)$ & & \\
\hline Baseline & $7.28 \pm 2.82$ & $6.41 \pm 1.25$ \\
\hline On Day 5 & $13.95 \pm 4.51^{\star \#}$ & $10.83 \pm 2.88^{\#}$ \\
\hline PLT count $\left(\times 10^{9} / L\right)$ & & \\
\hline Baseline & $260.75 \pm 61.97$ & $282.26 \pm 54.01$ \\
\hline On Day 5 & $302.70 \pm 74.42$ & $297.76 \pm 51.11$ \\
\hline
\end{tabular}

Note: Values represent means \pm standard deviation (SD)

* Compared to the I.V. HES group at the same period; \# Compared to the same group at baseline before ovarian

Haematocrit: HCT; White blood cell: WBC; platelet: PLT

Baseline: before ovarian stimulation

On Day 5: on the 5th day after oocyte retrieval

There were no statistically significant differences in coagulation and fibrinolytic parameters before ovarian stimulation between patients who received bromocriptine versus HES; only the activated partial thromboplastin time (APTT) was lower in the bromocriptine group than in the HES group on the 5th day after oocyte retrieval $(P<0.05)$. FIB, TT and D-D were significantly higher in both groups compared with their baseline levels (all $P<0.05$ ) (Table 5).

Table 5 Coagulation and fibrinolytic parameters at baseline and on the 5th day after oocyte retrieval 


\begin{tabular}{|lll|}
\hline Variables & Bromocriptine $(\boldsymbol{n}=\mathbf{5 2})$ & I.V HES $(\boldsymbol{n}=\mathbf{5 2})$ \\
\hline FIB $(\mathbf{g} / \mathrm{L})$ & & \\
\hline Baseline & $2.82 \pm 0.55$ & $2.83 \pm 0.58$ \\
\hline On Day 5 & $3.79 \pm 0.69^{\#}$ & $4.02 \pm 1.09^{\#}$ \\
\hline PT (s) & & \\
\hline Baseline & $11.17 \pm 0.70$ & $11.06 \pm 0.46$ \\
\hline On Day 5 & $10.95 \pm 0.56$ & $10.95 \pm 0.45$ \\
\hline PA (\%) & & \\
\hline Baseline & $112.48 \pm 17.46$ & $116.39 \pm 11.36$ \\
\hline On Day 5 & $119.74 \pm 12.70$ & $119.09 \pm 9.53$ \\
\hline TT (s) & & \\
\hline Baseline & $17.41 \pm 0.71$ & $17.76 \pm 1.09$ \\
\hline On Day 5 & $16.25 \pm 1.14^{\#}$ & $15.86 \pm 0.80^{\#}$ \\
\hline APTT (s) & & \\
\hline Baseline & $26.28 \pm 3.49$ & $26.53 \pm 2.68$ \\
\hline On Day 5 & $22.69 \pm 2.55^{\star \#}$ & $25.43 \pm 2.42$ \\
\hline D-D (mg/L) & & \\
\hline Baseline & $0.27 \pm 0.22$ & $1.83 \pm 1.29^{\#}$ \\
\hline On Day 5 & $1.91 \pm 1.46^{\#}$ & \\
\hline
\end{tabular}

Note: Values represent means \pm standard deviation (SD)

* Compared to the I.V. HES group at the same period; \# Compared to the same group at baseline before ovarian stimulation.

FIB: fibrinogen; PT: prothrombin time ; PA: plasminogen activator; TT: thrombin time; APTT: activated partial thromboplastin time; D-D: D-dimer.

Baseline: before ovarian stimulation

On Day 5: on the 5th day after oocyte retrieval

In assays for liver and kidney function, we found no significant difference between the two groups, either at baseline prior to ovarian stimulation or on the 5 th day after oocyte retrieval $(P>0.05)$. Both groups 
showed significantly higher transaminase activities, including ALT and AST, on the 5th day after oocyte retrieval compared with baseline $(P<0.05)$ (Table 6).

Table 6 Liver and renal function before ovarian stimulation and on the 5th day after oocyte retrieval in the two groups

\begin{tabular}{|c|c|c|}
\hline Variable & Bromocriptine $(n=52)$ & I.V HES $(n=52)$ \\
\hline \multicolumn{3}{|l|}{ ALT (U/L) } \\
\hline Baseline & $13.74 \pm 6.24$ & $15.70 \pm 13.50$ \\
\hline On Day 5 & $23.06 \pm 16.28^{\#}$ & $25.87 \pm 14.31^{\#}$ \\
\hline \multicolumn{3}{|l|}{ AST(U/L) } \\
\hline Baseline & $15.89 \pm 3.92$ & $16.14 \pm 4.97$ \\
\hline On Day 5 & $23.43 \pm 14.40^{\#}$ & $22.58 \pm 10.81^{\#}$ \\
\hline \multicolumn{3}{|l|}{ GGT(U/L) } \\
\hline Baseline & $16.38 \pm 7.73$ & $17.93 \pm 16.95$ \\
\hline On Day 5 & $23.29 \pm 22.30$ & $25.00 \pm 24.31$ \\
\hline \multicolumn{3}{|c|}{ BUN (mmol/L) } \\
\hline Baseline & $4.14 \pm 0.89$ & $3.88 \pm 0.91$ \\
\hline On Day 5 & $4.39 \pm 0.82$ & $4.14 \pm 0.78$ \\
\hline \multicolumn{3}{|c|}{$\mathrm{UA}(\mu \mathrm{mol} / \mathrm{L})$} \\
\hline Baseline & $298.83 \pm 75.93$ & $282.66 \pm 56.80$ \\
\hline On Day 5 & $265.92 \pm 63.08$ & $299.79 \pm 95.74$ \\
\hline \multicolumn{3}{|c|}{ CRE ( $\mu \mathrm{mol} / \mathrm{L})$} \\
\hline Baseline & $53.85 \pm 7.49$ & $53.75 \pm 8.19$ \\
\hline On Day 5 & $57.09 \pm 6.30$ & $56.10 \pm 6.93$ \\
\hline \multicolumn{3}{|c|}{ eGFR $\left(\mathrm{ml} / \mathrm{min} / 1.73 \mathrm{~m}^{2}\right)$} \\
\hline Baseline & $126.65 \pm 21.85$ & $128.08 \pm 22.74$ \\
\hline On Day 5 & $116.38 \pm 14.98$ & $120.69 \pm 19.53$ \\
\hline
\end{tabular}

Note: Values represent means \pm standard deviation (SD)

\# Compared to the same group at baseline before ovarian 
ALT: alanine aminotransferase; AST: aspartate aminotransferase; GGT: glutamyl transpeptidase; BUN: blood urea nitrogen; UA: uric acid; CRE: creatinine; eGFR: estimated glomerular filtration rate.

Baseline: before ovarian stimulation

On Day 5: on the 5th day after oocyte retrieval

In addition, the medication was well-tolerated by all patients in the study group, with no obvious side effects recorded in either the bromocriptine or HES groups.

\section{Discussion}

This retrospective study evaluated the effectiveness and physiological effects of rectal bromocriptine administration in OHSS prevention among high risk women undergoing IVF treatment. When compared with I.V. HES, a similar proportion of patients given bromocriptine experienced moderate OHSS, and in all cases the rectal bromocriptine was well-tolerated with no recorded adverse events, avoiding infection of vaginal administration after OPU. Overall, rectal administration of bromocriptine is an effective, safe, convenient, and relatively economical approach to OHSS prevention.

Estradiol levels and number of retrieved oocytes are the two most commonly used risk markers for OHSS during COS for IVF. A number of reports have shown that the rate of OHSS incidence increases along with elevated serum estradiol [15],[14, 16-18], although there is currently a lack of consensus on an appropriate threshold cut-off for estradiol-associated risk. By contrast, the number of oocytes retrieved following follicular aspiration can serve as a more precise marker for risk of OHSS development [19]. According to Papanikolaou et al. [20], a threshold of $\geq 18$ follicles $\geq 11 \mathrm{~mm}$ in diameter and/or serum estradiol $\geq 5000$ $\mathrm{pg} / \mathrm{ml}$ yielded an $83 \%$ sensitivity rate with a specificity of $84 \%$ for predicting severe OHSS cases. In some studies, the patients were included if $\geq 20$ oocytes could be retrieved and/or $E_{2}$ levels were $>3000 \mathrm{pg} / \mathrm{ml}$ on the day of hCG [21,22], which were the standards adopted for inclusion in our study.

Several recent innovative approaches have addressed the need to mitigate the risk and severity of OHSS in IVF/ICSI cycles [10]. These strategies for OHSS prevention include [23] individualized COS, cancelling of the cycle, coasting [4,24], individualizing the hCG trigger doses, or administration of a GnRH agonist for patients receiving $\mathrm{GnRH}$ antagonist $[13,25,26]$. In addition, aspirin in low doses has also been examined as a possible preventive measure for OHSS [27]. Other approaches include the use of I.V. fluids at the time of oocyte retrieval, such as albumin, $\operatorname{HES}[28,29]$, and calcium [30], as well as embryo cryopreservation for later transfer during an unstimulated cycle. Ultimately, the discovery that the proangiogenic cytokine VEGF functions as the primary contributor in OHSS pathogenesis, followed by the insight that VEGF activity could be modulated via dopamine, together, have resulted in the broad of DAs for prevention of OHSS-associated events and symptoms. Specifically, in vivo studies have shown that DAs post-transcriptionally regulate the secretion of VEGF in luteinised granulosa cells [31]. Previous research in a rat ovarian hyperstimulation model showed that administration of low-dose DA can inhibit VEGF-mediated vascular hyperpermeability but does not affect VEGF receptor 2-dependent luteal 
angiogenesis [32]. Notably, DAs can increase renal blood flow by affecting VEGF receptor, glomerular filtration, and sodium excretion, subsequently preventing development of ascites and facilitating cytokine secretion (e.g., VEGF), thus suggesting the potential utility of DAs in OHSS therapy [33].

Among DAs, cabergoline has been reported as a reliable prophylactic treatment to limit OHSS incidence and severity among high risk patients [9] [24, 34-40]. Bromocriptine has received attention for similar effects to cabergoline, although few clinical studies have explored its properties in the prevention of OHSS. Bromocriptine is an ergot alkaloid dopamine receptor agonist that is potent as an agonist of the dopamine-2 receptor, but also functions as a weak dopamine-1 receptor antagonist. An initial series of trials apparently show that bromocriptine is as effective as cabergoline against OHSS, but is considerably lower in cost [41]. These current studies all used $2.5 \mathrm{mg}$ bromocriptine daily, but vary in their starting time and duration of administration, with some beginning on the day of OPU [22, 42], and others on the day of hCG [43], and have a maximum duration spanning 16 days. In our study, bromocriptine was administered for only 5 days, and appeared to result in good prevention of OHSS. One study reported contradictory results in which bromocriptine did not affect OHSS incidence. However, this study used an exceptionally low sample size of only 14 patients and fresh embryos were used for transplantation [43].

I.V. HES is regarded as an effective approach in the reduction or attenuation of OHSS incidence among high risk patients(28), and is widely used among ART units for prevention of OHSS [13] [44]. Since HES administration can lead to significantly increased vascular system volume [45], its administration therefore may also be accompanied by elevated osmotic pressure and decreased blood viscosity. Furthermore, the inhibition of platelet aggregation induced by HES can also reduce blood coagulation [46], thus circumventing hypovolemia and hemoconcentration [47]. It also warrants mention that DAs inhibit the phosphorylation of VEGF receptor-2 $[32,48]$. The activity of bromocriptine in decreasing vascular permeability and concurrent reduction in VEGF production can at least partially explain its mechanism of inhibiting OHSS. In this retrospective study, we observed no significant differences in the incidence of mild ( $13.46 \%$ vs $15.38 \%$ ) and moderate ( $7.69 \%$ vs $5.77 \%$ ) OHSS between the bromocriptine and HES groups ( $P>0.05)$, and no patient in either group developed severe OHSS and late OHSS. Our results indicated that rectal administration of bromocriptine was as effective as HES in reducing OHSS. In particular, the WBC counts and HCT values were higher in the bromocriptine group on Day 5 after oocyte retrieval than in the HES group, while APTT was shorter. Our study thus indicated that coagulation in the bromocriptine group did not improve as strongly as in the HES group.

Transaminase ALT and AST levels were also higher on Day 5 after oocyte retrieval than at the baseline prior to ovarian stimulation in both two groups, however, the values were within the normal range. This increase in ALT and AST may be related to the rise in estradiol levels in the patients during COS, which aggravates the burden on the liver. However, all transaminase levels were in the normal range for both groups. In addition, we found no significant difference in renal function between the baseline and Day 5 post oocyte retrieval for either group. These results show that bromocriptine is sufficiently safe and does not appear to affect liver and renal function at these doses which are appropriate for OHSS prevention [43].

Page $11 / 17$ 
Disadvantages of bromocriptine include the known side effects of nausea, headaches, and orthostatic dysregulation [49]. In one study, $24 \%$ of the patients displayed transient gastrointestinal intolerance to DA treatment [50]. Notably, patients with OHSS may experience gastrointestinal symptoms including nausea and vomiting in the absence of treatment. Given that some clinical trials have used oral administration $[42,43]$, which may have resulted in substantial aggravation of gastrointestinal reactions for patients with high-risk of OHSS. We therefore opted for bromocriptine administration via rectal insertion to avoid these side effects. Moreover, the relatively low dosage used in this study was apparently well-tolerated by our sample population, more convenient for patients than I.V. delivery. The lower price of bromocriptine compared with HES also provides a cost-saving benefit that is also appreciated by patients.

\section{Conclusion}

The present study showed that rectal administration of $2.5 \mathrm{mg}$ bromocriptine daily for 5 days beginning on the day of oocyte retrieval was apparently as effective as I.V. HES for reducing OHSS in patients at high risk of developing OHSS during gonadotropin ovarian stimulation in the context of IVF/ICSI cycles.

The lack of reported side effects among subjects contributes to our conclusion that bromocriptine is safe at the doses tested here, and is more cost-effective and conveniently administered than HES. Thus, bromocriptine represents a new and promising alternative treatment approach for the prevention of OHSS. One limitation of this study was that no investigation of pregnancies were conducted after fresh embryo transfer, although future and ongoing studies will focus on this aspect of bromocriptine treatment for prevention of OHSS.

\section{Abbreviations}

OHSS: ovarian hyperstimulation syndrome., I.V. : intravenous., HES: hydroxyethyl starch., ART: assisted reproductive technology., COS: controlled ovarian hyperstimulation., IVF: in vitro fertilization., hCG: human chorionic gonadotropin., VEGF: vascular endothelial growth factor., DA: dopamine agonist., ICSI: intracytoplasmic sperm injection., $\mathrm{E}_{2}$ : estradiol., $\mathrm{rFSH}$ : recombinant follicle stimulating hormone., HPHMG: highly purified urinary menopausal gonadotropins., GnRH: gonadotrophin releasing hormone., BMI: body mass index., TVS: transvaginal ultrasound., WBC: white blood cell., PLT: platelet., HCT: hematocrit., S.D. : standard deviation., FSH: follicle stimulating hormone., LH: luteinizing hormone., AFC: antral follicle count., AMH: anti-Müllerian hormone., Gn: gonadotropin., OPU: ovum pick-up., FIB: fibrinogen.; PT: prothrombin time.; PA: plasminogen activator.; TT: thrombin time.; APTT: activated partial thromboplastin time.; D-D: D-dimer., ALT: alanine aminotransferase.; AST: aspartate aminotransferase.; GGT: glutamyl transpeptidase.; BUN: blood urea nitrogen.; UA: uric acid.; CRE: creatinine.; eGFR: estimated glomerular filtration rate.

\section{Declarations}

Acknowledgements 
We would like to thank all the participants and co-workers in Beijing Obstetrics and Gynecology Hospital for their assistance in this study. We express our appreciation to Dr. Zhaoxia Jia for data analyzing.

\section{Authors' contributions}

SYW conceived and designed the experiments. QLZ analyzed the data and wrote the manuscript. YMM and CWJ selected the OHSS according to patients' physical conditions and recorded all the basic information. XMB and YJL helped collect the data. The author(s) read and approved the final manuscript.

\section{Funding}

This work was supported by Beijing Municipal Administration of Hospitals Incubating Program (PX2019053), Beijing Administration of Traditional Chinese Medicine (JJ2018-06).

\section{Availability of data and materials}

The datasets used and/or analysed in this study are available from the corresponding author on reasonable request.

\section{Ethics approval and consent to participate}

Ethical approval was given by the medical ethics committee of Beijing Obstetrics and Gynecology Hospital, Capital Medical University. All procedures were performed in accordance with ethical standards developed by the institutional and national research committee. Informed consent for their clinical data to be used for research purposes was obtained from all participants.

\section{Consent for publication}

Not applicable.

\section{Competing interests}

The authors declare no competing interests.

\section{References}

1. Aboulghar MA, Mansour RT. Ovarian hyperstimulation syndrome: classifications and critical analysis of preventive measures. Hum Reprod Update. 2003;9(3):275-89.

2. Soares SR, Gomez R, Simon C, Garcia-Velasco JA, Pellicer A. Targeting the vascular endothelial growth factor system to prevent ovarian hyperstimulation syndrome. Hum Reprod Update. 2008;14(4):321-33.

3. Vloeberghs V, Peeraer K, Pexsters A, D'Hooghe T. Ovarian hyperstimulation syndrome and complications of ART. Best practice research Clinical obstetrics gynaecology. 2009;23(5):691-709. 
4. Gera PS, Tatpati LL, Allemand MC, Wentworth MA, Coddington CC. Ovarian hyperstimulation syndrome: steps to maximize success and minimize effect for assisted reproductive outcome. Fertil Steril. 2010;94(1):173-8.

5. Mourad S, Brown J, Farquhar C. Interventions for the prevention of OHSS in ART cycles: an overview of Cochrane reviews. Cochrane Database Syst Rev. 2017;1(1):Cd012103.

6. Nastri CO, Ferriani RA, Rocha IA, Martins WP. Ovarian hyperstimulation syndrome: pathophysiology and prevention. J Assist Reprod Genet. 2010;27(2-3):121-8.

7. Busso CE, Garcia-Velasco J, Gomez R, Alvarez C, Simon C, Pellicer A. Symposium: Update on prediction and management of OHSS. Prevention of OHSS-dopamine agonists. Reproductive biomedicine online. 2009; 19(1): 43-51.

8. Gomez R, Soares SR, Busso C, Garcia-Velasco JA, Simon C, Pellicer A. Physiology and pathology of ovarian hyperstimulation syndrome. Semin Reprod Med. 2010;28(6):448-57.

9. Youssef MA, van Wely M, Hassan MA, et al. Can dopamine agonists reduce the incidence and severity of OHSS in IVF/ICSI treatment cycles? A systematic review and meta-analysis. Hum Reprod Update. 2010;16(5):459-66.

10. Nelson SM. Prevention and management of ovarian hyperstimulation syndrome. Thromb Res. 2017;151(Suppl 1):61-s4.

11. Gokmen O, Ugur M, Ekin M, Keles G, Turan C, Oral H. Intravenous albumin versus hydroxyethyl starch for the prevention of ovarian hyperstimulation in an in-vitro fertilization programme: a prospective randomized placebo controlled study. Eur J Obstet Gynecol Reprod Biol. 2001;96(2):187-92.

12. Chalumeau C, Moreau J, Gatimel N, et al. Establishment and validation of a score to predict ovarian response to stimulation in IVF. Reprod Biomed Online. 2018;36(1):26-31.

13. Humaidan P, Quartarolo J, Papanikolaou EG. Preventing ovarian hyperstimulation syndrome: guidance for the clinician. Fertil Steril. 2010;94(2):389-400.

14. Mathur RS, Akande AV, Keay SD, Hunt LP, Jenkins JM. Distinction between early and late ovarian hyperstimulation syndrome. Fertil Steril. 2000;73(5):901-7.

15. Asch RH, Ivery G, Goldsman M, Frederick JL, Stone SC, Balmaceda JP. The use of intravenous albumin in patients at high risk for severe ovarian hyperstimulation syndrome. Hum Reprod. 1993;8(7):1015-20.

16. Navot D, Relou A, Birkenfeld A, Rabinowitz R, Brzezinski A, Margalioth EJ. Risk factors and prognostic variables in the ovarian hyperstimulation syndrome. Am J Obstet Gynecol. 1988;159(1):210-5.

17. Morris RS, Paulson RJ, Sauer MV, Lobo RA. Predictive value of serum oestradiol concentrations and oocyte number in severe ovarian hyperstimulation syndrome. Hum Reprod. 1995;10(4):811-4.

18. Chen CD, Wu MY, Chao KH, Chen SU, Ho HN, Yang YS. Serum estradiol level and oocyte number in predicting severe ovarian hyperstimulation syndrome. Journal of the Formosan Medical Association = Taiwan yi zhi. 1997;96(10):829-34. 
19. Graf MA, Fischer R, Naether OG, Baukloh V, Tafel J, Nuckel M. Reduced incidence of ovarian hyperstimulation syndrome by prophylactic infusion of hydroxyaethyl starch solution in an in-vitro fertilization programme. Hum Reprod. 1997;12(12):2599-602.

20. Papanikolaou EG, Pozzobon C, Kolibianakis EM, et al. Incidence and prediction of ovarian hyperstimulation syndrome in women undergoing gonadotropin-releasing hormone antagonist in vitro fertilization cycles. Fertil Steril. 2006;85(1):112-20.

21. Graf MA, Fischer R, Naether OG, Baukloh V, Tafel J, Nückel M. Reduced incidence of ovarian hyperstimulation syndrome by prophylactic infusion of hydroxyaethyl starch solution in an in-vitro fertilization programme. Hum Reprod. 1997;12(12):2599-602.

22. Sherwal V, Malik S, Bhatia V. Effect of bromocriptine on the severity of ovarian hyperstimulation syndrome and outcome in high responders undergoing assisted reproduction. Journal of human reproductive sciences. 2010;3(2):85-90.

23. Fiedler K, Ezcurra D. Predicting and preventing ovarian hyperstimulation syndrome (OHSS): the need for individualized not standardized treatment. Reprod Biol Endocrinol. 2012;10:32.

24. Garcia-Velasco JA. How to avoid ovarian hyperstimulation syndrome: a new indication for dopamine agonists. Reprod Biomed Online. 2009;18(Suppl 2):71-5.

25. Kol S, Solt I. GnRH agonist for triggering final oocyte maturation in patients at risk of ovarian hyperstimulation syndrome: still a controversy? J Assist Reprod Genet. 2008; 25(2-3): 63 - 6.

26. Griesinger G, Schultz L, Bauer T, Broessner A, Frambach T, Kissler S. Ovarian hyperstimulation syndrome prevention by gonadotropin-releasing hormone agonist triggering of final oocyte maturation in a gonadotropin-releasing hormone antagonist protocol in combination with a "freezeall" strategy: a prospective multicentric study. Fertil Steril. 2011; 95(6): 2029-33, 33.e1.

27. Várnagy A, Bódis J, Mánfai Z, Wilhelm F, Busznyák C, Koppán M. Low-dose aspirin therapy to prevent ovarian hyperstimulation syndrome. Fertil Steril. 2010;93(7):2281-4.

28. Aboulghar $\mathrm{M}$, Evers $\mathrm{JH}, \mathrm{Al}$-Inany $\mathrm{H}$. Intravenous albumin for preventing severe ovarian hyperstimulation syndrome: a Cochrane review. Hum Reprod. 2002;17(12):3027-32.

29. Aboulghar M. Symposium: Update on prediction and management of OHSS. Prevention of OHSS. Reproductive biomedicine online. 2009; 19(1): 33-42.

30. El-Khayat W, Elsadek M. Calcium infusion for the prevention of ovarian hyperstimulation syndrome: a double-blind randomized controlled trial. Fertil Steril. 2015;103(1):101-5.

31. Ferrero H, García-Pascual CM, Pellicer N, Simón C, Pellicer A, Gómez R. Dopamine agonist inhibits vascular endothelial growth factor protein production and secretion in granulosa cells. Reprod Biol Endocrinol. 2015;13:104.

32. Gomez R, Gonzalez-Izquierdo M, Zimmermann RC, et al. Low-dose dopamine agonist administration blocks vascular endothelial growth factor (VEGF)-mediated vascular hyperpermeability without altering VEGF receptor 2-dependent luteal angiogenesis in a rat ovarian hyperstimulation model. Endocrinology. 2006;147(11):5400-11. 
33. Beltrame AL, Serafini P, Motta EL, Soares Junior JM, Baracat EC. The effects of bromocriptine on VEGF, kidney function and ovarian hyperstimulation syndrome in in vitro fertilization patients: a pilot study. Gynecol Endocrinol. 2013;29(3):201-4.

34. Manno M, Tomei F, Marchesan E, Adamo V. Cabergoline: a safe, easy, cheap, and effective drug for prevention/treatment of ovarian hyperstimulation syndrome? Eur J Obstet Gynecol Reprod Biol. 2005;122(1):127-8.

35. Shaltout A, Shohyab A, Youssef MA. Can dopamine agonist at a low dose reduce ovarian hyperstimulation syndrome in women at risk undergoing ICSI treatment cycles? A randomized controlled study. Eur J Obstet Gynecol Reprod Biol. 2012;165(2):254-8.

36. Torabizadeh A, Vahidroodsari F, Ghorbanpour Z. Comparison of albumin and cabergoline in the prevention of ovarian hyperstimulation syndrome: A clinical trial study. Iranian journal of reproductive medicine. 2013;11(10):837-42.

37. Tehraninejad ES, Hafezi M, Arabipoor A, Aziminekoo E, Chehrazi M, Bahmanabadi A. Comparison of cabergoline and intravenous albumin in the prevention of ovarian hyperstimulation syndrome: a randomized clinical trial. J Assist Reprod Genet. 2012;29(3):259-64.

38. Carizza C, Abdelmassih V, Abdelmassih S, et al. Cabergoline reduces the early onset of ovarian hyperstimulation syndrome: a prospective randomized study. Reprod Biomed Online. 2008;17(6):751-5.

39. Jellad S, Haj Hassine A, Basly M, Mrabet A, Chibani M, Rachdi R. Vascular endothelial growth factor antagonist reduces the early onset and the severity of ovarian hyperstimulation syndrome. Journal of gynecology obstetrics human reproduction. 2017;46(1):87-91.

40. Naredi N, Talwar P, Sandeep K. VEGF antagonist for the prevention of ovarian hyperstimulation syndrome: Current status. Medical journal. Armed Forces India. 2014;70(1):58-63.

41. Wiwanitkit V. Prevention of ovarian hyperstimulation syndrome. Fertil Steril. 2011; 95(8): e65; author reply e6.

42. Spitzer D, Wogatzky J, Murtinger M, Zech MH, Haidbauer R, Zech NH. Dopamine agonist bromocriptine for the prevention of ovarian hyperstimulation syndrome. Fertil Steril. 2011;95(8):2742-4.e1.

43. Beltrame AL, Serafini P, Motta EL, Soares Júnior JM, Baracat EC. The effects of bromocriptine on VEGF, kidney function and ovarian hyperstimulation syndrome in in vitro fertilization patients: a pilot study. Gynecol Endocrinol. 2013;29(3):201-4.

44. König E, Bussen S, Sütterlin M, Steck T. Prophylactic intravenous hydroxyethyle starch solution prevents moderate-severe ovarian hyperstimulation in in-vitro fertilization patients: a prospective, randomized, double-blind and placebo-controlled study. Hum Reprod. 1998;13(9):2421-4.

45. Lazrove S, Waxman K, Shippy C, Shoemaker WC. Hemodynamic, blood volume, and oxygen transport responses to albumin and hydroxyethyl starch infusions in critically ill postoperative patients. Critical care medicine. 1980;8(5):302-6. 
46. Harke $H$, Thoenies R, Margraf I, Momsen W. [The influence of different plasma substitutes on blood clotting and platelet function during and after operations (author's transl)]. Anaesthesist. 1976;25(8):366-73.

47. Nastri CO, Teixeira DM, Moroni RM, Leitão VM, Martins WP. Ovarian hyperstimulation syndrome: pathophysiology, staging, prediction and prevention. Ultrasound in obstetrics gynecology: the official journal of the International Society of Ultrasound in Obstetrics Gynecology. 2015;45(4):377-93.

48. Saylan A, Arioz DT, Koken T, Dilek H, Saylan F, Yilmazer M. Prevention of ovarian hyperstimulation syndrome in a rat model: efficacy comparison between cabergoline and meloxicam. Acta Obstet Gynecol Scand. 2010;89(5):692-9.

49. Crosignani PG. Current treatment issues in female hyperprolactinaemia. Eur J Obstet Gynecol Reprod Biol. 2006;125(2):152-64.

50. Barbosa FR, Silva CM, Lima GA, et al. Prevalence of obstructive sleep apnea in patients with prolactinoma before and after treatment with dopamine agonists. Pituitary. 2014;17(5):441-9. 